\title{
Diagrammes de puissance conformes pour l'ingénierie de réservoir
}

\author{
Nicolas Flandrin ${ }^{1, a}$, Houman Borouchaki ${ }^{2}$ et Chakib Bennis ${ }^{1}$ \\ 1 Institut Français du Pétrole, 1 \& 4 avenue de Bois Préau, 92852 Rueil-Malmaison Cedex, France \\ 2 Université de Technologie de Troyes, 12 rue Marie Curie, BP 2060, 10010 Troyes Cedex, France
}

Reçu le 25 février 2005, accepté le 21 mars 2005

\begin{abstract}
Résumé - Cet article présente une nouvelle approche de type frontale permettant de générer un maillage hybride 3D en simulation de réservoirs pétroliers. Le maillage épouse les directions des écoulements aux abords des puits et permet d'atteindre une meilleure précision dans les calculs numériques. On insère dans le maillage structuré hexaédrique du réservoir un maillage local, radial et structuré autour de chaque puits. Des maillages déstructurés polyédriques, fondés sur les diagrammes de puissance, assurent la transition entre les maillages structurés.
\end{abstract}

Mots clés : Maillages hybrides / diagrammes de Voronoï et de puissance / triangulations de Delaunay et régulière

\begin{abstract}
Conforming power diagrams for reservoir engineering. This paper presents a new frontal approach to generate a 3D hybrid mesh in reservoir flow simulation. The mesh follows the flow directions around each well and allows to increase the accuracy in numerical simulations. In the hexaedral structured reservoir mesh, a local radial structured mesh is inserted around each well. Unstructured polyedral meshes based on power diagrams are used to connect the structured meshes together.
\end{abstract}

Key words: Hybrid meshes / Voronoï and power diagrams / Delaunay and regular triangulations

\section{Introduction}

Les nouvelles avancées technologiques en imagerie sismique 3D et en forage/production permettent aujourd'hui d'obtenir une image réaliste et fidèle de l'architecture interne du réservoir pétrolier et d'en optimiser la production en forant des puits complexes déviés en 3D avec plusieurs niveaux de ramification. Dans ce nouveau contexte technologique, la génération de maillage devient un élément crucial pour les simulateurs de réservoir pétrolier car elle permet de décrire la géométrie de la structure géologique étudiée au moyen d'une représentation en éléments discrets dans lesquels on effectue des simulations.

Les mailleurs standards actuels du marché, basés sur du maillage Corner Point Geometry (CPG) [1] ont déjà montré leurs limites. Leur caractère structuré facilite leur utilisation et leur exploitation mais ceci leur infère en même temps une rigidité ne permettant pas de représenter des géométries complexes.

D'autres approches ont été récemment proposées avec notamment les maillages de type PErpendicular

\footnotetext{
a Auteur correspondant : nicolas.flandrin@ifp.fr
}

BIssector (PEBI) [2] entièrement déstructurés qui sont obtenus en utilisant les diagrammes de Voronoï $[3,4]$. Ceux-ci possèdent plus de souplesse et ont permis d'obtenir des résultats prometteurs mais ils sont encore en $2,5 \mathrm{D}$ et leur manque de structure les rend très difficiles à exploiter.

Dans [5-7], une nouvelle approche hybride a été proposée en $2 \mathrm{D}$ puis étendue en $3 \mathrm{D}$ pour suivre la direction radiale des écoulements autour des puits. Cette approche allie les avantages des deux approches, structurée et déstructurée tout en limitant leurs désavantages. Ce maillage hybride est composé d'un maillage hexaédrique structuré décrivant la géométrie du réservoir, de maillages radiaux structurés s'adaptant localement à la direction des écoulements autour de chaque puits et de maillages polyédriques non structurés (basés sur l'utilisation de diagrammes de puissance $[8,9]$ ) assurant leur connexion.

Cet article présente une nouvelle approche de type frontale pour générer un tel maillage hybride en 3D. Dans une première partie, on présente les contraintes numériques imposées par les schémas numériques de type volumes finis et gouvernant la construction du maillage hybride. Ensuite, certaines définitions sont rappelées sur 
les notions de diagramme de puissance et de triangulation régulière. Finalement, on présente la nouvelle approche et les différentes étapes qui permettent de générer un maillage de transition conforme entre un maillage de puits et un maillage de réservoir en 3D.

\section{Données du problème}

Le modèle hybride proposé en 2D est composé de trois maillages élémentaires :

- une grille CPG (ou cartésienne non uniforme en 3D) structurée décrivant l'architecture du réservoir,

- des grilles radiales circulaires structurées s'adaptant localement à la direction des écoulements dans les aires de drainage de chaque puits et permettant d'atteindre une meilleure précision dans les calculs numériques,

- des maillages polyédriques non structurés, satisfaisant aux propriétés des volumes finis, assurant la transition entre les maillages structurés.

Alors que la génération des maillages structurés est bien connue en ingénierie de réservoir, leur connexion via l'utilisation de maillages de transitions est l'objet de nombreuses recherches actuelles [10]. Pour utiliser des schémas numériques de type volumes finis centrés sur les mailles, le maillage de transition doit respecter les contraintes suivantes :

- la convexité : chaque maille polygonale doit être convexe;

- la conformité : le maillage de transition doit être conforme aux arêtes (ou aux faces en 3D) situées sur la frontière de la cavité de sorte que le maillage global soit un maillage conforme;

- l'orthogonalité duale : la droite joignant les centres de deux mailles adjacentes doit être orthogonale à leur arête (ou face en 3D) commune afin d'utiliser des approximations à deux points dans les schémas numériques de type volumes finis;

- l'auto-centrage : les sites (ou centres) des mailles doivent être situés à l'intérieur de celles-ci.

Pour construire un maillage de transition répondant à ces critères, une méthode utilisant les diagrammes de puissance a été introduite en 2D dans [11] puis étendue à la 3D dans [7]. Généralisant la notion de diagramme de Voronoï, les diagrammes de puissances génèrent des cellules polyédriques convexes vérifiant la propriété d'orthogonalité duale et permettent de satisfaire la propriété de conformité dans le cas des réservoirs définis par des grilles CPG ou cartésiennes non uniformes (ce que l'on ne peut obtenir avec les diagrammes de Voronoï). Le but de ce travail est de présenter une nouvelle approche de type frontale permettant de générer un diagramme de puissance conforme entre les maillages de puits et de réservoir décrit par une grille cartésienne non uniforme en 3D.
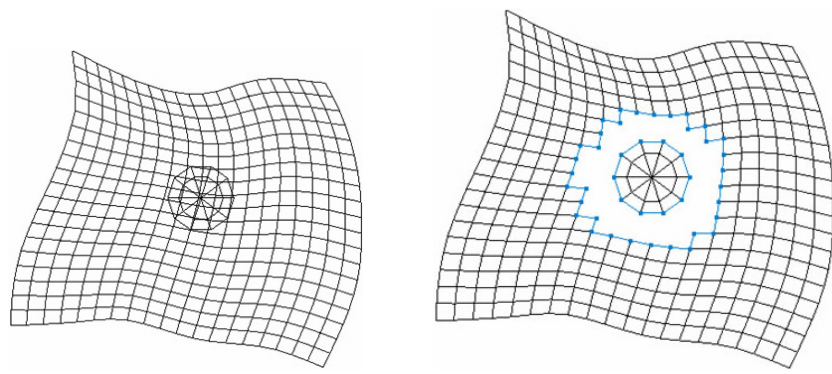

Fig. 1. Définition d'une cavité entre un puits et un réservoir.

\section{Rappel de la méthodologie utilisée en 2D}

On suppose donné un maillage de réservoir de type $\mathrm{CPG}$ dans lequel on insère un maillage de puits (Fig. 1 à gauche). Une fois les maillages superposés, on supprime (ou désactive) toutes les mailles du réservoir en contact et à proximité immédiate du puits (Fig. 1 à droite) de sorte qu'ensuite, aucune maille du puits ne chevauche une maille du réservoir. De la sorte, on définit une cavité entre le puits et le réservoir, l'espace à couvrir par le maillage de transition. Les arêtes de la frontière de la cavité sont ensuite extraites et le problème à résoudre consiste alors à construire un diagramme de puissance dont les cellules s'appuient exactement sur les arêtes de contrainte de la cavité.

Les étapes de construction du maillage de transition sont les suivantes :

- On commence par construire la triangulation de Delaunay [12] des sommets de la cavité (Fig. 2) en vérifiant que les arêtes de la cavité sont aussi des arêtes de la triangulation (condition nécessaire pour appliquer la méthode).

- Pour chaque arête de contrainte $a$ de la cavité, on choisit deux points pondérés $\left(P_{1}, \omega_{1}\right)$ et $\left(P_{2}, \omega_{2}\right)$ sur l'arête duale (ou arête de Voronoï obtenue via les triangles de la triangulation) associée à $a$, un à l'intérieur et l'autre à l'extérieur de la cavité (Fig. 2 au centre), le poids $\omega_{i}$ de ces sites étant défini par $\left\|\overrightarrow{P_{i} X}\right\|$ où $X$ est une extrémité de $a$.

- Finalement, on construit le diagramme de puissance ayant pour sites l'ensemble des sites internes à la cavité (Fig. 2 à droite).

Le diagramme de puissance obtenu est alors conforme au résultat souhaité : il est convexe, orthogonal et les arêtes de la cavité font partie intégrante du maillage de transition généré.

Cette méthodologie a été étendue à la $3^{\text {ème }}$ dimension dans [7] où on y explique comment on peut construire un diagramme de puissance 3D qui soit conforme aux faces quadrilatérales de la cavité. On y montre que si les sommets de ces faces sont cocycliques et coplanaires (ce qui est le cas des réservoirs décrits par une grille cartésienne non uniforme et des puits rectilignes décrits par une grille radiale circulaire) alors il est possible de construire un diagramme de puissance 3D qui s'appuie exactement dessus. Il suffit en effet de choisir, pour chaque quadrilatère $q$ de 

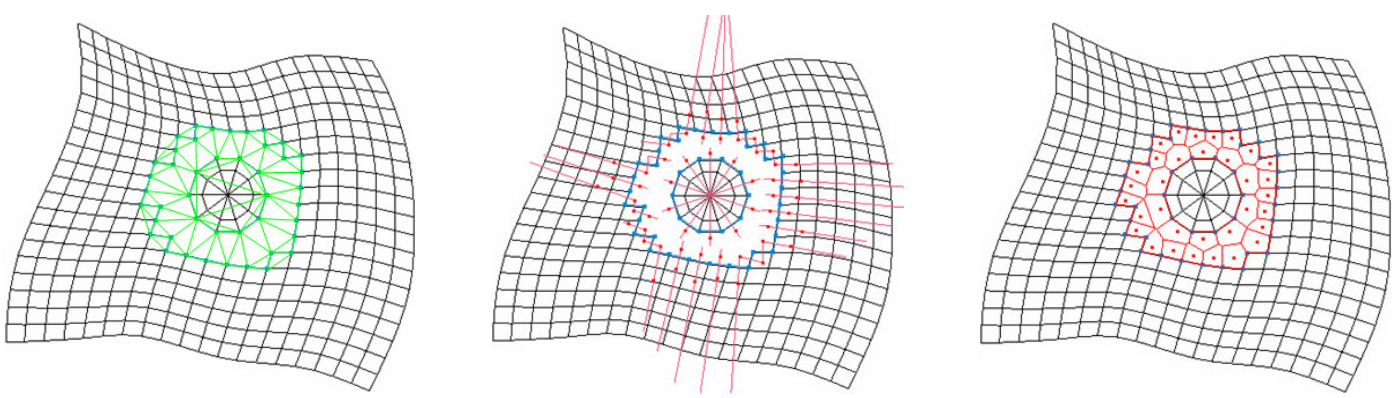

Fig. 2. Définition des sites de la cavité et construction du diagramme de puissance correspondant.

la cavité, deux sites $\left(P_{1}, \omega_{1}\right)$ et $\left(P_{2}, \omega_{2}\right)$ de part et d'autre de $q$ (un à l'intérieur et l'autre à l'extérieur de la cavité) tels que ces sites soient situés exactement à égale distance des sommets de $q$. En d'autres termes, $\left(P_{1}, \omega_{1}\right)$ et $\left(P_{2}, \omega_{2}\right)$ sont choisis sur l'arête duale de $q$ qui est en fait un segment de droite obtenu en reliant les centres des sphères circonscrites aux tétraèdres de Delaunay qui partagent une face triangulaire avec $q$ (si l'on décompose celui-ci en deux triangles distincts) : ce segment est orthogonal au plan défini par $q$ et passe par le centre du cercle circonscrit à $q$. Le poids des sites $\left(P_{1}, \omega_{1}\right)$ et $\left(P_{2}, \omega_{2}\right)$ est défini par $\left\|\overrightarrow{P_{i} X}\right\|$ où $X$ est une extrémité de $q$.

L'avantage de cette approche est qu'elle fonctionne également dans le cas des faces quadrilatérales non cocycliques présentes sur la frontière des puits déviés ou des réservoirs décrits par une grille CPG peu déformée. Dans ces deux cas, des problèmes de conformité apparaissent sur la frontière du maillage de transition généré. Toutefois ils peuvent être résolus en appliquant un procédé de correction qui consiste à bouger et à supprimer certains sommets présents sur la frontière de maillage de transition (cf. Sect. 5.5).

\section{Diagrammes de puissance et triangulations régulières}

L'objet de cette section est de rappeler la définition de certaines notions géométriques nécessaires à la bonne compréhension de cet article.

Point pondéré : Soit $P$ un point de $\mathbb{R}^{3}$ auquel on associe un poids $\omega$. On appelle point pondéré et on note $\rho$ le couple $(P, \omega)$. Notons au passage que $\rho$ peut aussi être interprété comme une sphère $\Sigma$ de centre $P$ et de rayon $\omega$.

Puissance : La puissance d'un point $X$ par rapport au point pondéré $\rho(P, \omega)$ ou par rapport à la sphère $\Sigma$ est définie par :

$$
\Pi(X, \rho)=\Pi(X, \Sigma)=\|\overrightarrow{P X}\|^{2}-\omega^{2}
$$

Produit de puissance : Le produit de puissance de deux points pondérés $\left(P_{i}, \omega_{i}\right)$ et $\left(P_{j}, \omega_{j}\right)$ est défini par :

$$
\Pi\left(\left(P_{i}, \omega_{i}\right),\left(P_{j}, \omega_{j}\right)\right)=\left\|\overrightarrow{P_{i} P_{j}}\right\|^{2}-\omega_{i}^{2}-\omega_{j}^{2}
$$

Notons que si le poids $\omega_{i}$ est égal à zéro, le produit de puissance $\Pi\left(\left(P_{i}, \omega_{i}\right),\left(P_{j}, \omega_{j}\right)\right)=\Pi\left(P_{i},\left(P_{j}, \omega_{j}\right)\right)$ est la puissance du point $P_{i}$ par rapport au point pondéré $\left(P_{j}, \omega_{j}\right)$. Les points pondérés $\left(P_{i}, \omega_{i}\right)$ et $\left(P_{j}, \omega_{j}\right)$ sont orthogonaux si leur produit de puissance est égal à zéro.

Plan radical : Le plan radical de deux points pondérés $\left(P_{i}, \omega_{i}\right)$ et $\left(P_{j}, \omega_{j}\right)$ est le plan défini par les points ayant la même puissance vis-à-vis de $\left(P_{i}, \omega_{i}\right)$ et de $\left(P_{j}, \omega_{j}\right)$. En particulier, si les sphères $\Sigma_{i}\left(P_{i}, w_{i}\right)$ et $\Sigma_{j}\left(P_{j}, w_{j}\right)$ ont une intersection non nulle, le plan radical est le plan passant par leur intersection.

Sphère de puissance : La sphère de puissance de quatre points pondérés $\left(P_{i}, \omega_{i}\right),\left(P_{j}, \omega_{j}\right),\left(P_{k}, \omega_{k}\right)$ et $\left(P_{l}, \omega_{l}\right)$ est l'unique sphère de puissance $(P, \omega)$ orthogonale à chacun de ces points pondérés. Les coordonnées du centre $P$ et la valeur de $\omega$ sont obtenues en résolvant le système suivant :

$$
\left\{\begin{array}{l}
\left\|\overrightarrow{P_{i} P}\right\|^{2}-\omega_{i}^{2}=\left\|\overrightarrow{P_{j} P}\right\|^{2}-\omega_{j}^{2} \\
\left\|\overrightarrow{P_{i} P}\right\|^{2}-\omega_{i}^{2}=\left\|\overrightarrow{P_{k} P}\right\|^{2}-\omega_{k}^{2} \\
\left\|\overrightarrow{P_{i} P}\right\|^{2}-\omega_{i}^{2}=\left\|\overrightarrow{P_{l} P}\right\|^{2}-\omega_{l}^{2} \\
\left\|\overrightarrow{P_{i} P}\right\|^{2}-\omega_{i}^{2}-\omega^{2}=0
\end{array}\right.
$$

Diagramme de puissance : Soit $\mathcal{S}=\left\{\rho_{i} \ldots \rho_{n}\right\}$ un ensemble de points pondérés. Le diagramme de puissance de $\mathcal{S}$ est l'ensemble des cellules $V_{i}$ définies par :

$$
V_{i}=\left\{X \in \mathbb{R}^{3}, \Pi\left(\rho_{i}, X\right) \leq \Pi\left(\rho_{j}, X\right), \forall \rho_{j} \in \mathcal{S}, j \neq i\right\}
$$

Chaque cellule correspond à un point pondéré $\left(P_{i}, \omega_{i}\right)$ de $\mathcal{S}$ et est le lieu des points $P \in \mathbb{R}^{3}$ dont la puissance par rapport à $\left(P_{i}, \omega_{i}\right)$ est plus petite que sa puissance par rapport à n'importe quel autre point $\left(P_{j}, \omega_{j}\right)$ de $\mathcal{S}$. Le diagramme de puissance étend la notion de diagramme de Voronoï puisqu'un diagramme de Voronoï n'est autre qu'un diagramme de puissance pour lequel les pondérations de tous les sites sont égales. Il est à noter cependant qu'un site peut ne pas avoir de cellule (ou pour être plus exact avoir une cellule vide) dans le diagramme de puissance de $\mathcal{S}$. On peut montrer toutefois que, si pour tout couple $\left(\rho_{i}, \rho_{j}\right)$ de points pondérés de $\mathcal{S}$ la relation $\left|\omega_{i}^{2}-\omega_{j}^{2}\right|<\left\|\overrightarrow{P_{i} P_{j}}\right\|^{2}$ est satisfaite, alors toutes les cellules de $\mathcal{S}$ sont non vides et auto-centrées.

À titre d'illustration, la figure 3 à gauche montre le diagramme de Voronoï associé au nuage de points 

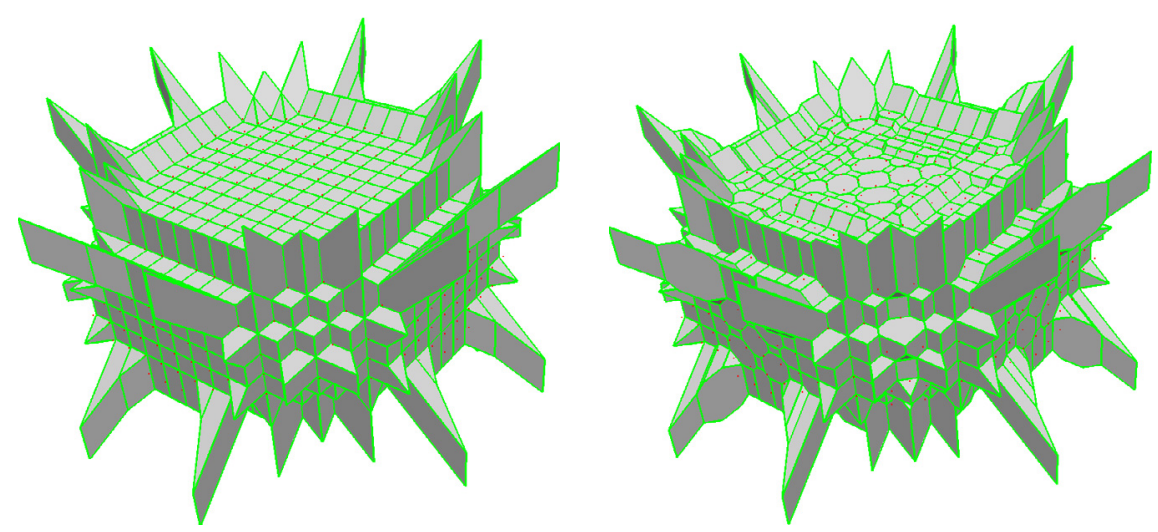

Fig. 3. Diagramme de Voronoï (à gauche) et diagramme de puissance (à droite).
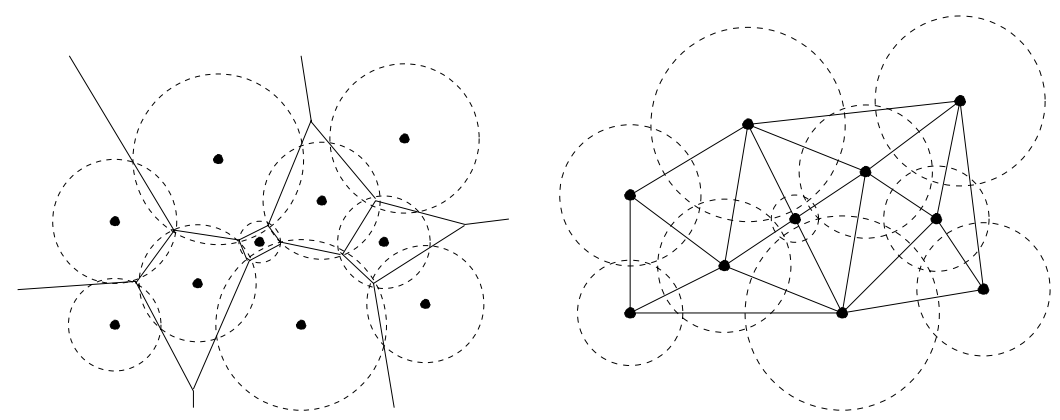

Fig. 4. Diagramme de puissance (à gauche) et triangulation régulière correspondante (à droite).

$(i, j, k)$ à coordonnées entières défini par les relations $-10 \leq i, j, k \leq 10$ et $i^{2}+j^{2}+k^{2}<100$. En affectant un poids plus important aux points se trouvant sur les plans diagonaux et en s'assurant que la triangulation régulière correspondant existe, on obtient le diagramme de puissance illustré par la figure 3 à droite.

Une présentation détaillée des diagrammes de puissance et des exemples de leur utilisation pourront être trouvés dans $[3,9,13]$.

Triangulation régulière : Plaçons-nous dans la situation où chaque cellule $V_{i}$ du diagramme de puissance est une région non vide associée à un site $\rho_{i}$ de $\mathcal{S}$. À partir des $V_{i}$, le dual peut être construit pour former la triangulation régulière [14]. En particulier, les faces des cellules ont une puissance égale vis-à-vis des deux points qu'elles séparent, définissant ainsi les plans radicaux des arêtes de la triangulation. Autrement dit, on obtient la triangulation cherchée (ou plus exactement les arêtes de celle-ci) en joignant les sommets de $\mathcal{S}$ qui appartiennent à deux cellules adjacentes (Fig. 4). La triangulation obtenue est unique et se compose de tétraèdres, dans le cas où les points de $\mathcal{S}$ sont en position générale ${ }^{1}$. Dans le cas contraire, des éléments non simpliciaux sont construits qui peuvent être ultérieurement subdivisés en tétraèdres (donnant ainsi lieu à plusieurs triangulations pour un même jeu de points).

\footnotetext{
${ }^{1}$ Un ensemble de sites est dit en position générale s'il n'existe pas de configuration où plus de quatre sites ont une puissance égale vis-à-vis d'un même point $X \in \mathbb{R}^{3}$.
}

La triangulation régulière étend la notion de triangulation de Delaunay puisqu'une triangulation de Delaunay n'est autre qu'une triangulation régulière pour laquelle les pondérations de tous les sites sont égales.

\section{Construction du maillage de transition}

On présente ici une nouvelle approche que l'on pourrait qualifier de frontale permettant de générer un maillage de transition conforme en 3D. Elle diffère de la méthode décrite en 3 par le fait que la triangulation de Delaunay des sommets de la cavité n'est plus nécessaire pour déterminer les sites du diagramme de puissance recherché. En effet, comme on va le voir dans cette section, les mailles du puits et du réservoir présentes de chaque côté de la frontière de la cavité permettent de les définir.

\subsection{Définition de la cavité}

La définition de la cavité, tout comme le choix des sites, est une étape importante de la méthodologie car elle détermine l'espace à couvrir par le maillage de transition. Du point de vue de la visualisation, de l'exploration ou encore de l'interprétation, elle doit être de taille minimale pour limiter la zone non structurée du maillage hybride mais elle doit aussi être de taille suffisante pour permettre de construire un diagramme de puissance conforme entre le puits et le réservoir. Pour construire une telle cavité, on introduit un coefficient de dilatation locale $\alpha$ (fonction de 

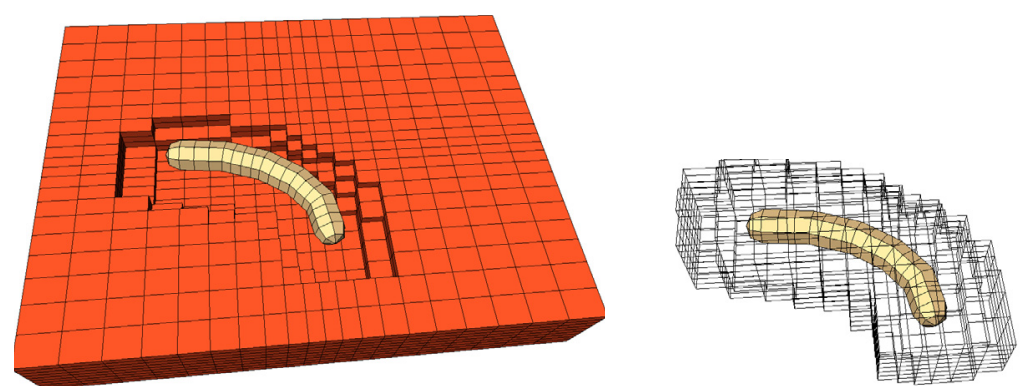

Fig. 5. Cavité définie entre un maillage de puits et un maillage de réservoir en 3D.

la taille locale des mailles du puits et du réservoir) et on dilate le puits en fonction de ce coefficient. On désactive alors l'ensemble des mailles du réservoir ayant une intersection non nulle avec l'image du puits dilaté (Fig. 5 à gauche). La valeur du coefficient de dilatation $\alpha$ est alors :

$$
\alpha=1+\frac{\delta_{\mathrm{P}}+\delta_{\mathrm{R}}}{r_{\mathrm{d}}}
$$

où $r_{\mathrm{d}}$ est le rayon de drainage du puits, $\delta_{\mathrm{P}}$ est la taille d'arête maximale du puits et $\delta_{\mathrm{R}}$ est la taille d'arête locale du réservoir.

On extrait finalement la frontière de la cavité qui est constituée exclusivement de faces quadrilatérales et de triangles aux extrémités du puits. Elle est composée de deux fronts : un provenant du réservoir et l'autre provenant du puits (Fig. 5 à droite).

\subsection{Choix des sites}

Dans [11] et [7], une triangulation de Delaunay des sommets de la cavité est utilisée pour positionner les sites nécessaires à la construction d'un diagramme de puissance conforme entre les maillages du puits et du réservoir. Les sites sont définis sur les arêtes duales des faces de la cavité ce qui leur permet d'être situés à égale distance des sommets des faces quadrilatérales associées (si toutefois les sommets de ces faces sont coplanaires et cosphériques). Cette distance est différente pour chaque site et est fonction de l'espace présent entre la frontière du puits et celle du réservoir qui est donné localement par les simplexes de la triangulation.

Cependant, puisque la taille de la cavité est connue et maîtrisée, cette triangulation de Delaunay n'est pas nécessaire : il suffit en effet de définir chaque site assez éloigné de la frontière de la cavité tel que sa distance à chacun des sommets des faces de contrainte associées soit la même.

\subsubsection{Méthodologie générale}

On propose ici une nouvelle approche qui consiste à définir la position de chaque site à partir des mailles du réservoir et du puits partageant au moins une face avec la frontière de la cavité.

\section{Choix des sites associés au réservoir}

Soit $\mathcal{S}_{\mathrm{R}}$ l'ensemble des mailles du réservoir partageant au moins une face avec la frontière de la cavité, alors :

- pour chaque maille inactive $\in \mathcal{S}_{\mathrm{R}}$, on propose un site interne à la cavité qui est le centre de la sphère circonscrite (cf. Sect. 5.2.2) à cette maille (Fig. 6 à gauche),

- pour chaque maille active $\in \mathcal{S}_{\mathrm{R}}$, on définit un site externe à la cavité qui est le centre de la sphère circonscrite à cette maille (Fig. 6 à droite).

\section{Choix des sites associés au puits}

On utilise ici un raisonnement similaire. Soit donc $\mathcal{S}_{\mathrm{P}}$ l'ensemble des mailles du puits partageant au moins une face avec la frontière de la cavité, alors, pour chaque maille active $\in \mathcal{S}_{\mathrm{P}}$ :

- on propose un site externe à la cavité qui est le centre de la sphère circonscrite (cf. Sect. 5.2.2) à cette maille (Fig. 7 à gauche) ;

- on construit une maille virtuelle en ajoutant autour du puits une couche de mailles supplémentaire de taille $\delta_{\mathrm{P}}$ où $\delta_{\mathrm{P}}$ est la taille d'arête maximale du puits. Un site interne à la cavité est alors défini par le centre de la sphère circonscrite à cette maille virtuelle (Fig. 7 à droite).

\section{Rassemblement des sites}

L'ensemble des sites nécessaires à la construction du diagramme de puissance est obtenu en rassemblant tous les sites internes et externes à la cavité définis au niveau du réservoir et au niveau du puits (Fig. 8). Les sites internes à la cavité seront les centres des cellules du diagramme de puissance et les sites externes à la cavité permettront d'assurer leur conformité avec la frontière de la cavité.

On notera que chaque site (interne ou externe) est associé à une ou plusieurs faces de la cavité appelé(e)s face(s) de contrainte associée(s) au site et que chaque face de la cavité est toujours associée à deux sites : un site interne et un site externe à la cavité. Par ailleurs, le poids $\omega$ de chaque site $(P, \omega)$ est égal à la distance moyenne de $P$ à un des sommets de sa (ou ses) face(s) de contrainte. 

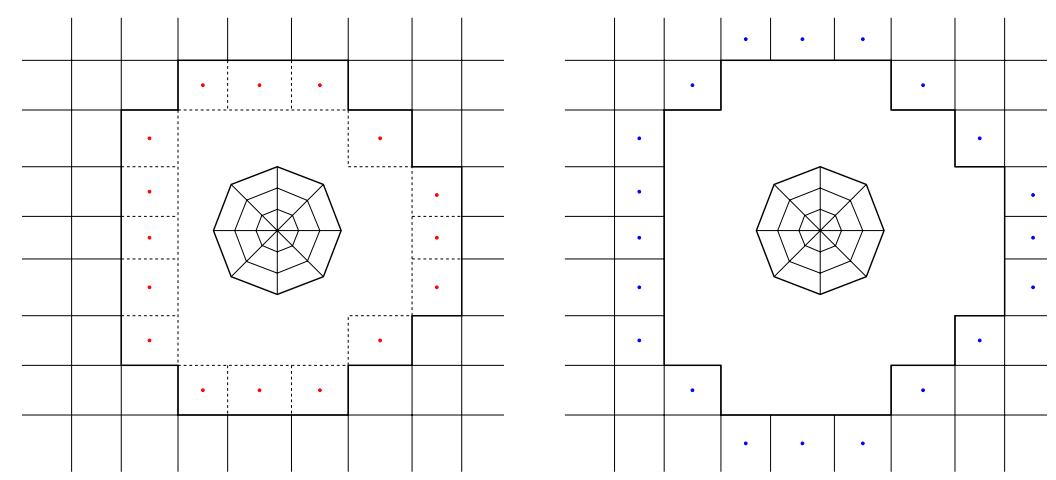

Fig. 6. Schéma représentant le choix des sites internes (à gauche) et celui des sites externes (à droite) associés au réservoir.
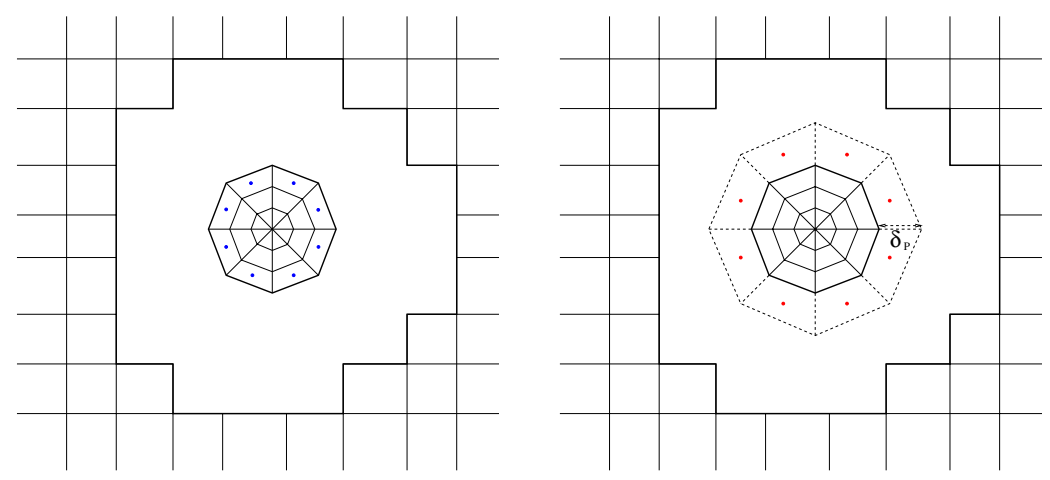

Fig. 7. Schéma représentant le choix des sites externes (à gauche) et celui des sites internes (à droite) associés au puits.
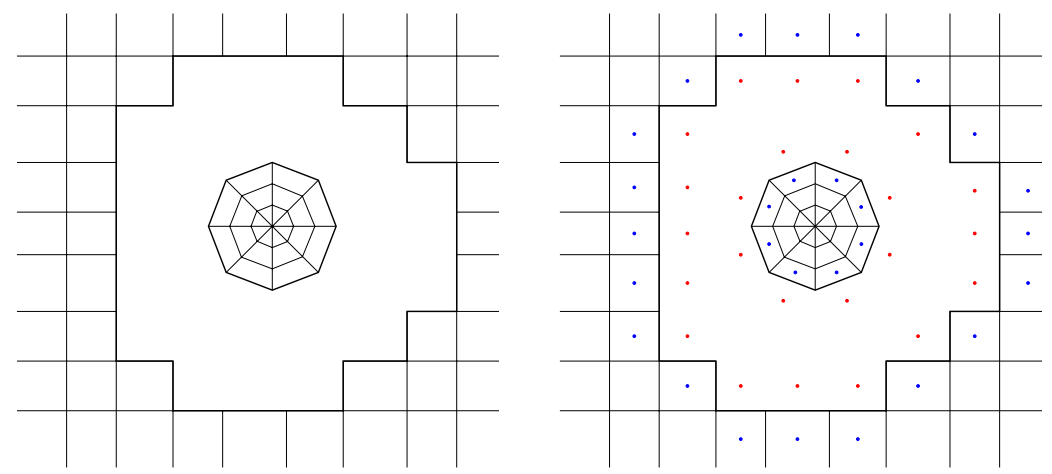

Fig. 8. Schéma récapitulant le choix de tous les sites nécessaires à la construction d'un diagramme de puissance conforme.

\subsection{2 Évaluation du centre de la sphère circonscrite à un hexaèdre}

On décrit ici la définition du centre de la sphère circonscrite à une maille hexaédrique quelconque à l'aide d'un calcul de distance et de la résolution du système linéaire associé par une approximation au sens des moindres carrés (le centre de la sphère circonscrite à une maille hexaédrique parallélépipédique étant égal au point milieu d'une des grandes diagonales de la maille).

Soient $P_{i}(i=1 \ldots 8)$ les 8 sommets d'une maille hexaédrique quelconque. On cherche à définir la sphère de centre $P$ passant par (ou au plus près de) chacun des 8 sommets de cette maille (Fig. 9).

En exprimant la distance entre $P$ et $P_{1}$ et en imposant que cette distance doit être égale à celle entre $P$ et chacun
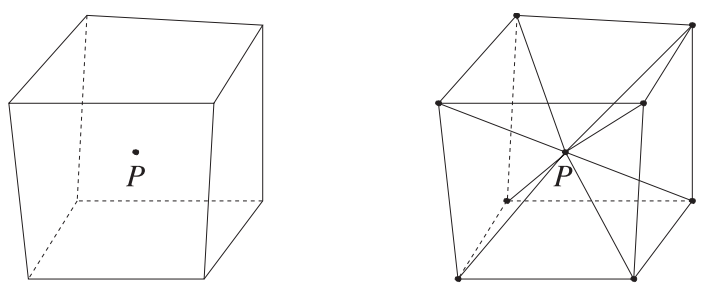

Fig. 9. Évaluation du centre de la sphère circonscrite à un hexaèdre.

de 7 autres sommets $P_{i}(i=2 \ldots 8)$, on obtient la relation suivante :

$$
\begin{gathered}
\left\|\overrightarrow{P P_{1}}\right\|=\left\|\overrightarrow{P P_{i}}\right\| \\
\sqrt{{ }^{t} \overrightarrow{P P_{1}} \overrightarrow{P P_{1}}}=\sqrt{t \overrightarrow{P P_{i}} \overrightarrow{P P_{i}}}
\end{gathered}
$$


ou encore, en développant et en élevant le tout au carré :

$$
\begin{aligned}
&\left(x_{i}-x_{1}\right) x+\left(y_{i}-y_{1}\right) y+\left(z_{i}-z_{1}\right) z= \\
& \frac{\left(x_{i}^{2}-x_{1}^{2}\right)+\left(y_{i}^{2}-y_{1}^{2}\right)+\left(z_{i}^{2}-z_{1}^{2}\right)}{2}
\end{aligned}
$$

avec les notations : $\overrightarrow{P P_{i}}=\left(\begin{array}{c}x_{i} \\ y_{i} \\ z_{i}\end{array}\right)$ et $P=\left(\begin{array}{c}x \\ y \\ z\end{array}\right)$.

On a alors un système sur-déterminé de la forme :

$$
A P=B
$$

où $A$ est une matrice $7 \times 3$ fonction de $\left(x_{i}, y_{i}, z_{i}\right)$ et $B$ un vecteur à 7 lignes :

$$
\begin{gathered}
A=\left(\begin{array}{cc}
\left(x_{2}-x_{1}\right) x\left(y_{2}-y_{1}\right) y\left(z_{2}-z_{1}\right) z \\
\vdots & \vdots \\
\left(x_{8}-x_{1}\right) x & \left(y_{8}-y_{1}\right) y\left(z_{8}-z_{1}\right) z
\end{array}\right) \\
B=\frac{1}{2}\left(\begin{array}{c}
\left(x_{2}^{2}-x_{1}^{2}\right)+\left(y_{2}^{2}-y_{1}^{2}\right)+\left(z_{2}^{2}-z_{1}^{2}\right) \\
\vdots \\
\left(x_{8}^{2}-x_{1}^{2}\right)+\left(y_{8}^{2}-y_{1}^{2}\right)+\left(z_{8}^{2}-z_{1}^{2}\right)
\end{array}\right)
\end{gathered}
$$

Pour résoudre un tel système sur-déterminé et tenter de satisfaire toutes les équations simultanément (ce qui est impossible dans le cas général), on utilise une approximation au sens des moindres carrés, c'est-à-dire qu'on essaie de minimiser l'écart entre les vecteurs $A P$ et $B$ de $\mathbb{R}^{6}$ en minimisant le carré de la norme euclidienne de leur différence. La formule générale pour approcher cette solution consiste à obtenir un système bien déterminé (de 3 équations à 3 inconnues) en multipliant l'expression précédente à gauche par ${ }^{t} A$ :

$$
{ }^{t} A A P={ }^{t} A B
$$

Ce système est résolu par une méthode classique, par exemple celle du pivot Gauss. Le point $P$ ainsi trouvé est le centre de la sphère qui passe au plus près des 8 sommets de la maille hexaédrique considérée.

\subsubsection{Ajustement des sites}

Comme on vient de le voir, chaque site construit à partir d'une maille hexaédrique quelconque est le centre de la sphère qui passe au plus près des 8 sommets de cette maille. Sa distance vis-à-vis de ces 8 sommets n'est donc pas la même ce qui peut, dans certains cas, poser des problèmes de conformité importants. Or, on sait que chaque site est associé à une ou plusieurs faces de la cavité et que, pour être conforme, celui-ci doit être situé à égale distance des sommets (au nombre de 4 à 8) de cette ou de ces faces. On propose donc de bouger itérativement ce site dans l'espace pour minimiser l'écart maximal entre les distances de ce site à chacun de ces sommets de contrainte (Fig. 10).

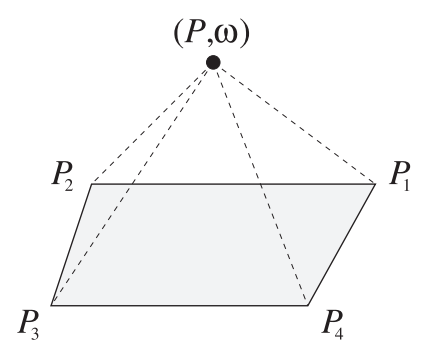

Fig. 10. Ajustement du site $(P, \omega)$.

Soit $(P, \omega)$ un site donné et soit $P_{1}, P_{2}, P_{3}$ et $P_{4}$ les sommets de la face quadrilatérale associée, l'algorithme de bougé consiste à :

1. Calculer la distance moyenne $l_{\text {moy }}$ de $P$ à chacun des sommets $P_{i}$ :

$$
l_{\mathrm{moy}}=\frac{\sum_{i=1}^{4}\left\|\overrightarrow{P_{i} P}\right\|}{4}
$$

2. Définir le point $Q$ tel que :

$$
Q=\frac{\sum_{i=1}^{4}\left(P_{i}+l_{\text {moy }} \frac{\overrightarrow{P_{i} P}}{\left\|\overrightarrow{P_{i} P}\right\|}\right)}{4}
$$

3. Si $Q$ est différent de $P$ (c'est-à-dire si $\left|x_{Q}-x_{P}\right|>\varepsilon$ ou $\left|y_{Q}-y_{P}\right|>\varepsilon$ ou $\left|z_{Q}-z_{P}\right|>\varepsilon$ où $\varepsilon$ est la précision souhaitée), remplacer $P$ par $Q$ et aller en 2 .

À la convergence de l'algorithme, le site $(P, \omega)$ a atteint une position d'équilibre. En remplaçant $P$ par le point $Q$ et en définissant $\omega$ comme étant la distance moyenne de $P$ à chaque sommet $P_{i}$, on minimise encore un peu plus les problèmes de conformité que l'on abordera dans la section 5.5.

\subsection{Correction des sites}

À ce stade, on a défini un ensemble de sites internes et externes à la cavité qui garantissent l'existence d'un diagramme de puissance 3D qui est conforme aux faces quadrilatérales de la cavité (si toutefois les sommets de ces faces sont coplanaires et cocycliques). Cependant, bien qu'un tel diagramme satisfasse les conditions d'orthogonalité duale et de convexité relatives aux schémas numériques de type volumes finis, il se peut que certaines cellules soient vides ou non auto-centrées. En effet, un site peut très bien être en dehors de sa cellule de puissance ou bien, ce qui est encore plus grave, un site peut ne pas avoir de cellule. Cela est dû à la définition des sites qui a été faite de manière indépendante. Chaque site a été déterminé en fonction des mailles du puits et $\mathrm{du}$ réservoir sans tenir compte de son interaction avec les autres sites. Or, pour garantir la construction d'un diagramme de puissance qui est constitué de cellules non vides et auto-centrées, on doit prendre en compte cette interaction mutuelle des sites. On propose donc d'effectuer 


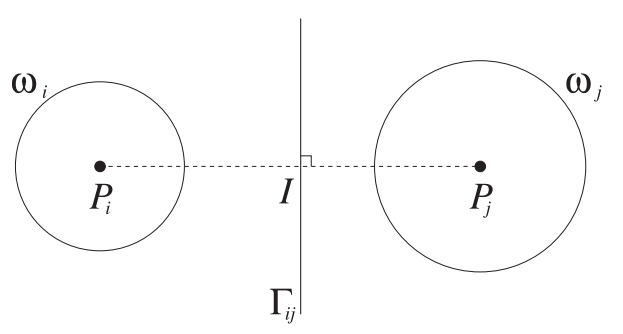

Fig. 11. Plan radical de deux points pondérés.

une étape supplémentaire dite de correction qui consiste à corriger la position de certains sites via la correction de leur poids.

\subsubsection{Condition d'auto-centrage}

Soient deux sites $\left(P_{i}, \omega_{i}\right)$ et $\left(P_{j}, \omega_{j}\right)$ et soit $\Gamma_{i j}$ leur plan radical, c'est-à-dire le lieu d'égale puissance de $P_{i}$ et de $P_{j}$. Pour que les cellules de puissance associées à $\left(P_{i}, \omega_{i}\right)$ et $\left(P_{j}, \omega_{j}\right)$ soient auto-centrées, ces deux sites doivent être situés de part et d'autre de $\Gamma_{i j}$ (Fig. 11).

Soit $I$ le point d'intersection du plan radical $\Gamma_{i j}$ et du segment $\left[P_{i} P_{j}\right]$ :

$$
I=t P_{i}+(1-t) P_{j} \quad \text { avec } \quad t \in[0,1]
$$

Puisque $I$ est sur le plan radical $\Gamma$, on peut écrire l'égalité des puissances de $\Pi\left(I,\left(P_{i}, \omega_{i}\right)\right)$ et de $\Pi\left(I,\left(P_{j}, \omega_{j}\right)\right)$ :

$$
\left\|\overrightarrow{P_{i} I}\right\|^{2}-\omega_{i}^{2}=\left\|\overrightarrow{P_{j} I}\right\|^{2}-\omega_{j}^{2}
$$

Les équations (13) et (14) permettent alors d'exprimer $t$ en fonction de $\left(P_{i}, \omega_{i}\right)$ et de $\left(P_{j}, \omega_{j}\right)$ :

$$
\begin{aligned}
\left\|\overrightarrow{P_{i} I}\right\|^{2}-\omega_{i}^{2} & =\left\|\overrightarrow{P_{j} P_{i}}+\overrightarrow{P_{i} I}\right\|^{2}-\omega_{j}^{2} \\
\left\|\overrightarrow{P_{i} I}\right\|^{2}-\omega_{i}^{2} & =\left\|\overrightarrow{P_{j} P_{i}}\right\|^{2}+2 \overrightarrow{P_{j} P_{i}} \cdot \overrightarrow{P_{i} I}+\left\|\overrightarrow{P_{i} I}\right\|^{2}-\omega_{j}^{2} \\
-\omega_{i}^{2} & =\left\|\overrightarrow{P_{j} P_{i}}\right\|^{2}+2 \overrightarrow{P_{j} P_{i}} \cdot(1-t) \overrightarrow{P_{i} P_{j}}-\omega_{j}^{2} \\
-\omega_{i}^{2} & =(2 t-1)\left\|\overrightarrow{P_{i} P_{j}}\right\|^{2}-\omega_{j}^{2} \\
\hookrightarrow \quad t & =\frac{\left\|\overrightarrow{P_{i} P_{j}}\right\|^{2}-\omega_{i}^{2}+\omega_{j}^{2}}{2\left\|\overrightarrow{P_{i} P_{j}}\right\|^{2}}
\end{aligned}
$$

Or, puisque $t \in[0,1]$ :

$$
0 \leq \frac{\left\|\overrightarrow{P_{i} P_{j}}\right\|^{2}-\omega_{i}^{2}+\omega_{j}^{2}}{2\left\|\overrightarrow{P_{i} P_{j}}\right\|^{2}} \leq 1
$$

D'où :

$$
\left|\omega_{i}^{2}-\omega_{j}^{2}\right| \leq\left\|\overrightarrow{P_{i} P_{j}}\right\|^{2}
$$

Cette condition est la condition nécessaire et suffisante exprimant le fait que le plan radical $\Gamma_{i j}$ coupe le segment $\left[P_{i} P_{j}\right]$.

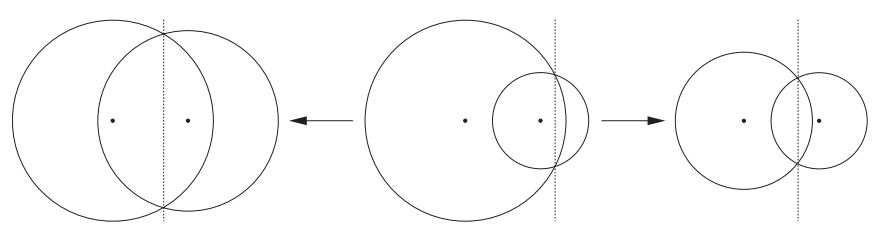

Fig. 12. Déplacement du plan radical par modification du poids d'un site.

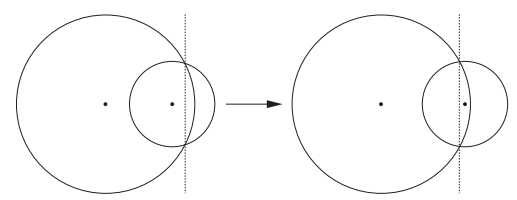

Fig. 13. Déplacement du plan radical par déplacement d'un site.

\subsubsection{Correction de deux sites ne vérifiant pas la propriété d'auto-centrage}

Soient deux sites $\left(P_{i}, \omega_{i}\right)$ et $\left(P_{j}, \omega_{j}\right)$ tels que $\mid \omega_{i}^{2}-$ $\omega_{j}^{2} \mid>\left\|\overrightarrow{P_{i} P_{j}}\right\|^{2}$. Cela implique que ces sites se situent du même côté de leur plan radical $\Gamma_{i j}$ et donc que l'un de ces sites, au moins, n'est pas dans sa cellule du diagramme de puissance.

Pour que le site situé en dehors de sa cellule se retrouve à l'intérieur, il faut s'employer à ce que le plan radical $\Gamma_{i j}$ se retrouve entre les deux sites. Pour cela, deux solutions sont envisageables. La première solution consiste à modifier le poids d'un des sites, c'est-à-dire à modifier le rayon de la sphère associée à ce site (en l'augmentant si le poids de celui-ci est le plus petit des deux, ou en le diminuant dans le cas contraire). Ceci est illustré par la figure 12.

La seconde possibilité consiste simplement à déplacer le site. En effet, déplacer un site modifie la position de l'intersection des sphères associées aux sites et donc la position de leur plan radical (Fig. 13).

Or, dans la situation qui nous intéresse, le poids d'un site est lié à sa position. En effet, le poids $\omega$ d'un site, localisé au point $P$, est tel que $\omega$ correspond à la distance moyenne entre $P$ et les extrémités du (ou des) quadrilatère(s) de la cavité associé(s) au site. On ne peut donc pas modifier sa puissance sans le déplacer, ni le déplacer sans modifier son poids. On propose donc ici une combinaison de ces deux manières de procéder.

Pour chaque quadrilatère $q$ de la frontière de la cavité, deux sites sont définis sur son arête duale de part et d'autre de $q$. Les sites étant choisis de manière arbitraire sur cette arête duale, il est tout à fait possible de les déplacer le long de celle-ci (Fig. 14).

Considérons de nouveau les sites $\left(P_{i}, \omega_{i}\right)$ et $\left(P_{j}, \omega_{j}\right)$ tels que $\left|\omega_{i}^{2}-\omega_{j}^{2}\right|>\left\|\overrightarrow{P_{i} P_{j}}\right\|^{2}$. Pour que leur plan radical $\Gamma_{i j}$ coupe le segment $\left[P_{i} P_{j}\right]$, on doit modifier la position et le poids d'un des deux sites. En pratique, on considère le site dont le poids est le plus grand, par exemple $\left(P_{i}, \omega_{i}\right)$, et on le déplace le long de son arête duale en le rapprochant de son quadrilatère de contrainte et en mettant à jour son poids jusqu'à ce que la condition (17) soit vérifiée (Fig. 15). 


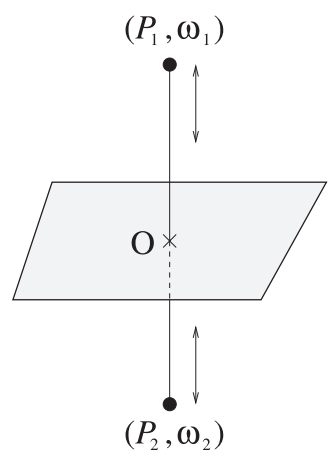

Fig. 14. Degré de latitude sur le déplacement des sites.

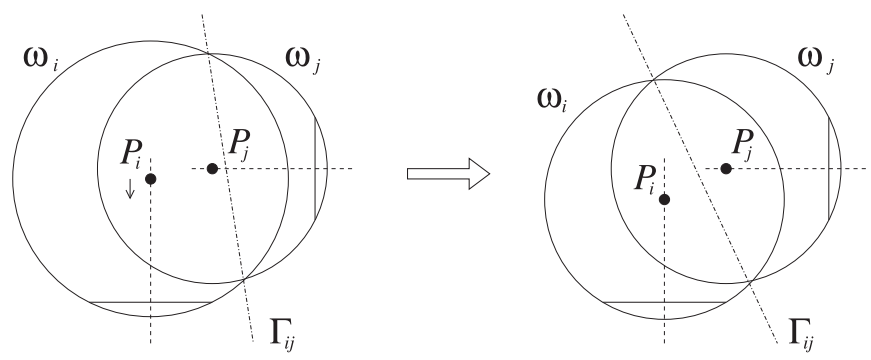

Fig. 15. Déplacement d'un plan radical par déplacement d'un site et modification de son poids.

\subsubsection{Algorithme de correction des sites}

On présente ici un algorithme itératif qui consiste à vérifier la condition (17) en chacun des sites de la cavité. Si cette condition n'est pas vérifiée par certains d'entre eux, on les bouge suivant la méthode décrite dans la section précédente. Plus en détails, l'algorithme utilisé est le suivant :

1. On initialise le nombre de corrections $n$ à zéro.

2. Pour chaque site de la cavité $\left(P_{i}, \omega_{i}\right)$, on recherche l'ensemble des sites $\left(P_{j}, \omega_{j}\right), j \neq i$ tels que $\left\|\overrightarrow{P_{i} P_{j}}\right\|^{2} \leq$ $\omega_{i}^{2}$ (on utilise une méthode de localisation par bucketing [15] pour trouver ces sites).

3. Pour chaque couple de sites $\left(\left(P_{i}, \omega_{i}\right),\left(P_{j}, \omega_{j}\right)\right)$, on calcule la différence $\left|\omega_{i}^{2}-\omega_{j}^{2}\right|$. Si cette différence est supérieure à $\left\|\overrightarrow{P_{i} P_{j}}\right\|^{2}$, le plan radical n'est pas situé entre $P_{i}$ et $P_{j}$; une correction doit donc être faite; on incrémente $n$. Si on note $(P, \omega)$ le site ayant le poids le plus important, on déplace $P$ le long de son arête duale en le rapprochant de son quadrilatère de contrainte d'une distance de $x \%$ (par exemple $10 \%$ ), $\omega$ étant mis à jour par la même occasion.

4. Si $n>0$, on retourne en 1 .

À la convergence de cet algorithme, les sites de la cavité vérifient tous la condition d'auto-centrage. Un diagramme de puissance convexe, orthogonal, conforme et auto-centré peut donc être construit à partir de ces sites.

\subsection{Construction du diagramme de puissance}

À ce niveau, on est capable de construire le maillage de transition souhaité. Pour cela, on commence par calculer la triangulation régulière des sites de la cavité en utilisant une méthode incrémentale [16]. Puis, par dualité, on obtient le diagramme de puissance recherché (Fig. 16) ou plus exactement les arêtes de celui-ci en joignant deux à deux les centres de puissances des tétraèdres adjacents.

\subsection{Problèmes de conformité}

Le diagramme de puissance généré est orthogonal, auto-centré et théoriquement conforme aux faces quadrilatérales de la cavité (si toutefois les sommets de ces faces sont cosphériques et coplanaires). Cependant, à cause de problèmes de précision numérique et de la présence de faces non cocycliques sur la frontière du puits (et donc l'impossibilité de définir des sites qui soient situés exactement à égale distance des sommets de ces faces), cette conformité n'est pas assurée partout. Une procédure est alors utilisée pour la rétablir en identifiant les faces externes du maillage de transition avec les faces de la cavité (par bougé et suppression de sommets). En particulier, si $V$ est une maille de transition donnée, l'algorithme de correction mis en place est le suivant :

1. On commence par déterminer l'ensemble $\mathcal{A}=$ $\left\{A_{1} \ldots A_{n}\right\}(1<n \leq 8)$ des sommets des quadrilatères de contrainte associés à $V$.

2. On détermine ensuite l'ensemble $\mathcal{B}=\left\{B_{1} \ldots B_{m}\right\}$ (avec $m \geq n$ ) des sommets des faces libres ${ }^{2}$ de $V$.

3. Finalement, on remplace chaque sommet $B_{i} \in \mathcal{B}(1 \leq$ $i \leq m)$ par le sommet $A_{j} \in \mathcal{A}$ le plus proche :

$$
B_{i}=\left\{A_{j} \in \mathcal{A},\left\|\overrightarrow{B_{i} A_{j}}\right\| \leq\left\|\overrightarrow{B_{i} A_{k}}\right\|, \forall A_{k} \in \mathcal{A}, k \neq j\right\}
$$

En appliquant cette procédure à toutes les cellules du diagramme de puissance, on obtient le maillage de transition conforme souhaité (Fig. 17) en mettant à jour toute la structure de données : on supprime les points multiples au sein d'une même face et on détruit les faces dont le nombre de sommets est inférieur à 3 .

\section{Résultats}

La méthode proposée dans ce papier permet d'insérer un ou plusieurs puits dans un même réservoir. Aussi, lorsque deux ou plusieurs puits sont proches, les cavités correspondantes peuvent fusionner et donner naissance à un unique maillage de transition. La figure 18 illustre le cas d'un maillage hybride où trois puits radiaux circulaires sont insérés dans une grille réservoir cartésienne non uniforme. Les trois grilles radiales sont alors connectées au maillage de réservoir grâce à l'utilisation de deux maillages de transition.

Par ailleurs, lorsque l'espace entre deux puits n'est pas suffisant pour construire un maillage de transition conforme, certaines mailles de ces puits sont désactivées

\footnotetext{
${ }^{2}$ Une face libre est une face qui n'est partagée que par une seule cellule de transition.
} 

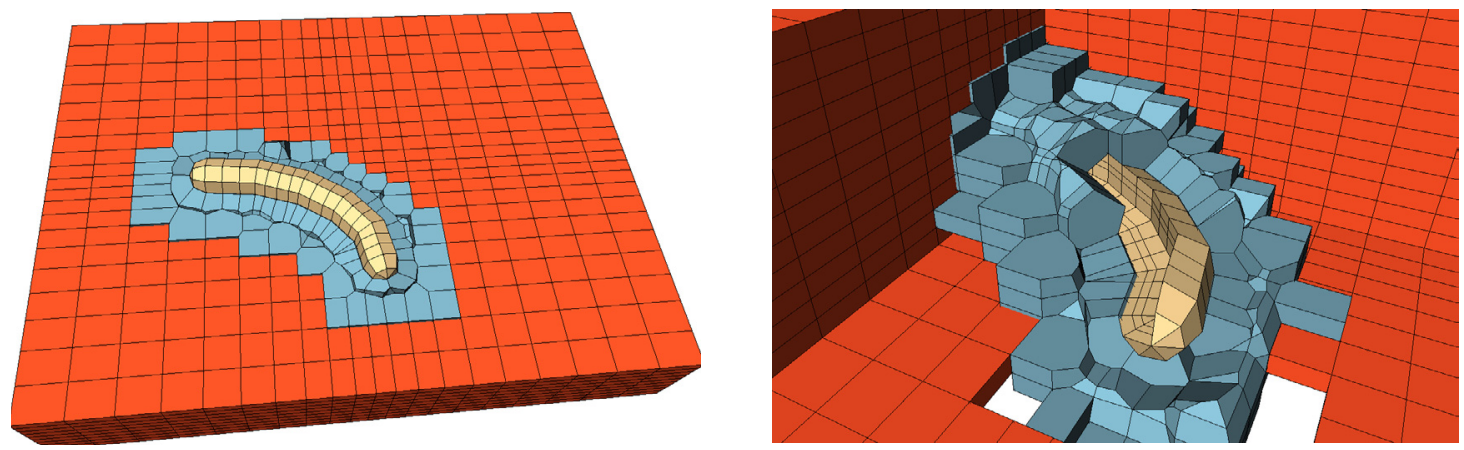

Fig. 16. Maillage de transition obtenu entre un maillage de puits et un maillage de réservoir en 3D.
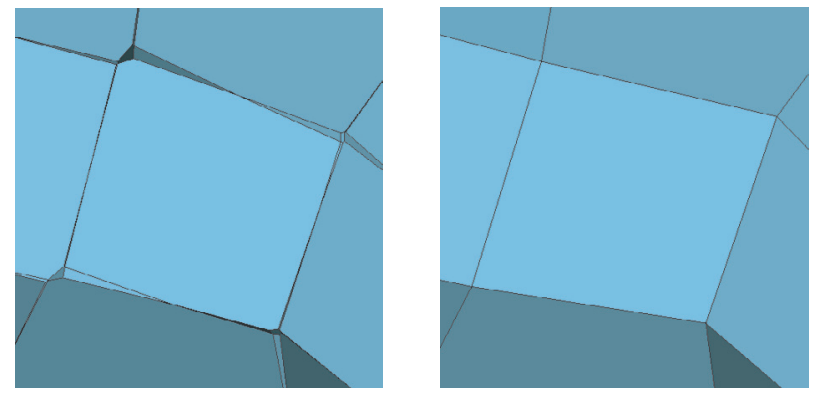

Fig. 17. Maillage de transition rendu conforme.

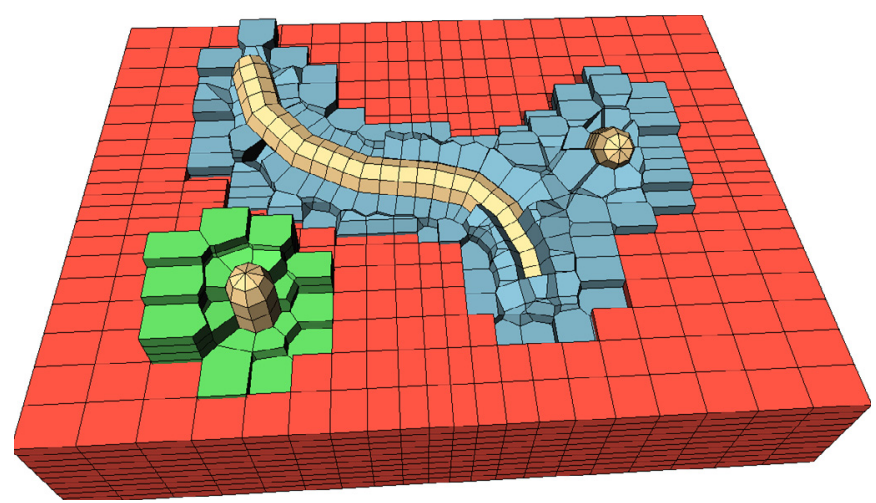

Fig. 18. Trois puits insérés dans un maillage de réservoir.

pour agrandir la taille de la cavité (Fig. 19) qui doit être de taille supérieure ou égale à $\delta_{1}+\delta_{2}$ où $\delta_{1}$ et $\delta_{2}$ sont les tailles d'arêtes maximales de ces deux puits.

\section{Conclusion}

Dans cet article, on a présenté une nouvelle méthode permettant de générer un diagramme de puissance conforme entre un réservoir décrit par une grille cartésienne non uniforme et les aires de drainage des puits représentées par des maillages radiaux circulaires.

La méthode proposée est très robuste lorsque les faces de la cavité sont coplanaires et cosphériques. Cependant, elle ne peut être appliquée telle quelle dans le cas des vrais réservoirs CPG à cause de la trop grande déformation des

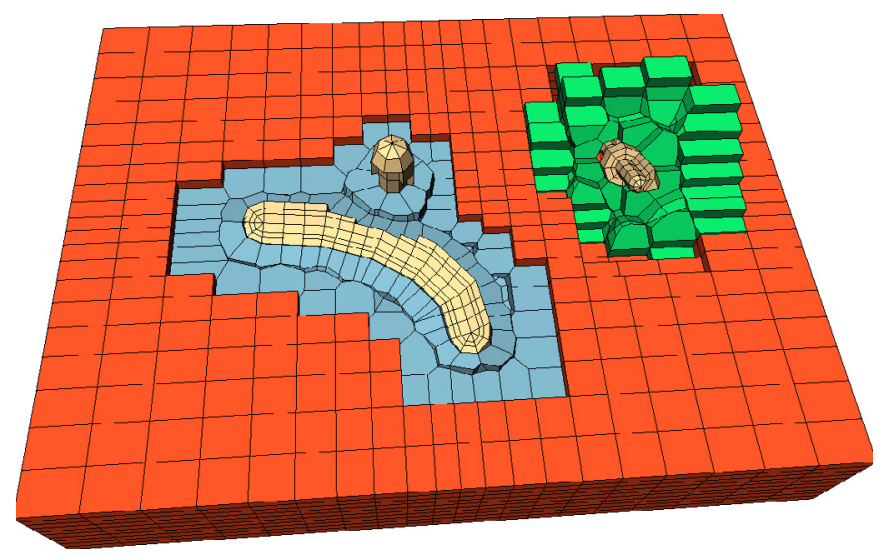

Fig. 19. Maillage de réservoir avec désactivation de certaines mailles de puits trop proches.

mailles. Une future extension possible de ces travaux est de considérer la géométrie des grilles CPG en utilisant des métriques anisotropes. Cela demande d'étendre l'ensemble des procédures proposées, en particulier la triangulation régulière et la construction de son dual, au cas Riemannien anisotrope.

\section{Références}

[1] D.K. Ponting, Corner point geometry in reservoir simulation, In Clarendon Press, editor, Proc. 1st European Conference on the Mathematics of Oil Recovery, Cambridge, 1989, pp. 45-65

[2] Z.E. Heinemann, C.W. Brand, Gridding techniques in reservoir simulation, In First and Second International Forum on Reservoir Simulation, Alpbach, Austria, September 1988-1989, pp. 339-426

[3] F. Aurenhammer, Voronoï diagrams: a survey of a fundamental geometric data structure, ACM Computing Surveys 23(3) (1991) 345-405

[4] S. Fortune, Voronoï diagrams, Delaunay triangulations, E.J. Goodman, J. O'Rourke (ed.), Handbook of Discrete and Computational Geometry, CRC Press LLC, 1997, chapter 20, pp. 377-388

[5] S. Balaven, C. Bennis, J.D. Boissonnat, S. Sarda, Generation of hybrid grids using power diagrams, In 7th International Conference on Numerical Grid Generation in Computational Field Simulations, 2000 
[6] S. Balaven-Clermidy, Génération de maillages hybrides pour la simulation des réservoirs pétroliers, Ph.D. thèse, École des mines de Paris, 2001

[7] N. Flandrin, H. Borouchaki, C. Bennis, 3d hybrid mesh generation for reservoir flow simulation, In 13th International Meshing Roundtable, Williamsburg, Virginia, USA, september 2004

[8] F. Aurenhammer, Power diagrams : properties, algorithms and applications, SIAM J. Comput. 16(1) (1987) $78-96$

[9] H. Imai, M. Iri, K. Murota, Voronoï diagrams in the Laguerre geometry and its applications, SIAM J. Comput. 14(1) (1985) 69-96

[10] O.A. Pedrosa, K. Aziz, Use of hybrid grid in reservoir simulation, In Proceedings of SPE Middle East Oil Technical Conference, Bahrain, March 1985, SPE, pp. 99-112
[11] S. Balaven, C. Bennis, J.D. Boissonnat, M. Yvinec, Conforming orthogonal meshes, In 11th International Meshing Roundtable, Ithaca, New York, USA, September 2002

[12] P.L. George, H. Borouchaki, Triangulation de Delaunay et maillage, Hermès, 1997

[13] J.D. Boissonnat, M. Yvinec, Géométrie Algorithmique, Ediscience International, 1995

[14] H. Edelsbrunner, N.R. Shah, Incremental topological flipping works for regular triangulations, Algorithmica 15 (1987) 223-241

[15] P.J. Frey, P.L. George, Maillages : applications aux éléments finis, Hermès, 1999

[16] H. Borouchaki, N. Flandrin, C. Bennis, Diagramme de Laguerre, C.R. Acad. Sci. Paris, à paraître 